\title{
Alternative methods to derive the Black-Scholes-Merton equation
}

\author{
Nattakorn Phewchean, Renato Costa, Masnita Misiran, Yongwimon Lenbury
}

Received: October 28, 2020. Revised: November 17, 2020, Accepted: November 19, 2020. Published: November 20, 2020.

\begin{abstract}
We investigate the derivation of option pricing involving several assets following the Geometric Brownian Motion (GBM). First, we propose some derivations based on the basic ideas of the assets. Next, we consider the trivial case where we have $n$ assets. Finally, we consider different drifts, volatilities and Wiener processes but now from $n$ stochastic assets taking into account a fixed-income.
\end{abstract}

Keywords-Black-Scholes-Merton equation, fixed income, option pricing, stochastic asset

\section{INTRODUCTION}

$\mathrm{O}$ PTION pricing is one of the mathematical processes in the financial market that has been utilized in the investment analysis. Derivative is a financial security that has the prices based on the other underlying assets; consequently, the mathematical approach of option pricing derivation can be applied in many perspectives. Option is one of the derivatives that the researchers have been investigating as an approach of evaluation. Fisher Black and Myron Scholes have proposed a new aspect of derivative pricing in 1973 [1]. This well-known Black-Scholes equation with Geometric Brownian Motion (GBM) has altered the way researchers view derivative-pricing both in terms of practice and theory [2]. Subsequently, the Black-Scholes equation has been applied to European option pricing by considering some essential variables such as stock price, strike price, interest rate and maturity time [3].

The derivations of Black-Scholes equation have been discussed for decades by many researchers. As a result, Robert Merton proposed the model extension for Black-Scholes equation which has taken into account the dividend yield as a factor for the mathematical model. In this way, the option

This work was financially supported by Mahidol University and the Centre of Excellence in Mathematics, CHE, Thailand.

N. Phewchean is with the Department of Mathematics, Faculty of Science, Mahidol University, Rama 6 Rd., Bangkok 10400, Thailand and is with the Centre of Excellence in Mathematics, PERDO, Thailand. (e-mail: nattakorn.phe@mahidol.ac.th).

R. Costa was with the Department of Mathematics, Faculty of Science, Mahidol University, Rama 6 Rd., Bangkok 10400, Thailand. (e-mail: noldo22@gmail.com).

M. Misiran. is with the School of Quantitative Sciences, UUM College of Arts and Sciences, Universiti Utara Malaysia, 06010 UUM Sintok, Kedah Darul Aman, Malaysia (e-mail: masnita.misiran@gmail.com).

Y. Lenbury is with the Department of Mathematics, Faculty of Science, Mahidol University, Rama 6 Rd., Bangkok 10400, Thailand and is with the Centre of Excellence in Mathematics, PERDO, Thailand. (corresponding author phone: (662) 201-5448; e-mail: yongwimon.len@mahidol.ac.th). pricing model has been improved, providing more accurate results for investors. The different ways to derive the BlackScholes formula lead to different types of actual practices, for instance by replicating the option with underlying bonds or stocks. Consequently, many alternative derivations of the Black-Sholes-Merton model have been proposed in research works such as derivations that apply the capital asset pricing model (CAPM), arbitrage pricing or risk neutral pricing method [4-6].

In order to have the best practice of the Black-Scholes-Merton equation, understanding of how to derive the Black-ScholesMerton equation in various perspectives is critical. In this paper, we propose some different perspectives of the BlackScholes-Merton model derivation. We investigate the pricing of Options formula involving several assets following the Geometric Brownian Motion (GBM). Firstly, we propose some derivations based on the basic ideas of the assets. Secondly, we consider the trivial case where we have $n$ assets. Finally, we consider the model derivation taking into account the dividend yield. Specifically, the aim of this research is to propose the Black-Scholes-Merton equation with $n$ stochastic assets and Black-Scholes-Merton model with the proposed extension of $n$ stochastic assets perspective with fixed income.

\section{II.FORMULA BACKGROUND}

The central idea is that the Black-Scholes model may be derived by understanding the basic concept of replicating portfolio by considering a bond and a stock. It can be shown that the returns of this replicating portfolio are equivalent to the option payoffs. However, by having the Black-Scholes equation, some big assumptions are required for the option pricing model derivation such as:

1. There is no arbitrage opportunity.

2. There is a constant risk-free interest rate.

3. Short selling allowance is included.

4. Efficient market is assumed.

5. A geometric Brownian motion is assumed.

6. There are no dividends.

The derivation of the Black-Scholes model can be carried out by the basic concept of investment such as the change of investment value in a very tiny time interval can be compared to the return value of the investment during that short time. Thus, it can be implied mathematically that 
$d V=r V d t$

which is

$\frac{d V}{V}=r d t$

where $V$ is the amount of investment and $r$ is the rate of return. By letting $M=\ln V$ and inserting the stochastic term of $\sigma \sqrt{\Delta t} d W(t)$, we have the following stochastic differential equation

$d M=r d t+\sigma \sqrt{\Delta t} d W(t)$

where $\sigma$ is volatility and $W(t)$ is the Wiener process. The mean and standard deviation of a stochastic term of $\sigma \sqrt{\Delta t} d W(t)$ are zero and $\sqrt{\Delta t}$ respectively. In this case of general replication, we can derive the Black-Scholes model by applying Ito's lemma and Taylor's theorem. Now, if we consider the portfolio that is represented by the bond $B(t)$ and the stock $S(t)$ then the option of $P(S(\mathrm{t}), t)$ can be priced by the models of this portfolio. From (2), we can write

$$
\frac{d B(t)}{B(t)}=r d t \text {. }
$$

The stock change, derived in the same way as (3), is

$$
d S(t)=\mu S(t) d t+\sigma S(t) d W(t) .
$$

Then, the option price, $P(S(\mathrm{t}), t)$, which depends on the stock value $S(t)$, by this method can be derived as

$$
\begin{aligned}
& d P(S(t), t)=\left(P(S(t), t)_{t}+\mu P(S(t), t)_{S} S(t)\right. \\
& \left.+\frac{1}{2} \sigma^{2} S(t)^{2} P(S(\mathrm{t}), t)_{S S}\right) d t+\sigma P(S(t), t)_{S} S(t) d W(t)
\end{aligned}
$$

This can lead to the way option pricing is done by having the Black-Scholes formula. In fact, there are various methods to derive the Black-Scholes equation. With this method, by assuming self-financing with the basic concept of portfolio replication, the option is replicated by bonds and stocks. By different perspective of derivation, the option pricing can be obtained by the different aspects of data such as the way to replicate the bond with option and stock instead or to replicate the stock with option and bond. In such a way, the BlackScholes model can be derived [5].

\section{N STOCHASTIC ASSETS}

Specifically, in this work, a generalization of Black-Scholes model derivation is proposed under the extension of $n$ stochastic assets perspective. By this concept, the derivative price depends on the $n$ uncorrelated underlying assets such as commodities, bonds, stocks, currencies, and so on. In reality, these assets can be continued as both deterministic and stochastic assets. However, in this paper, we suppose that a risk-free bond $B(\mathrm{t})$ is deterministic and the other assets $S_{i}(t)$ are stochastic assets with the Wiener process. Since the derivative price can depend on various types of assets, it can be shown mathematically that

$P(S(t), t)=\left(x_{1} S_{1}(t)+x_{2} S_{2}(t)+x_{3} S_{3}(t)+\ldots\right.$

$\left.+x_{n} S_{n}(t)\right)+y B(t)$

where $x_{i}$ and $y$ are the unit numbers of assets. Next, we obtain

$$
\begin{aligned}
& d P(S(t), t)=\left(x_{1} d S_{1}(t)+x_{2} d S_{2}(t)+x_{3} d S_{3}(t)\right. \\
& \left.+\ldots+x_{n} d S_{n}(t)\right)+y d B(t)
\end{aligned}
$$

Again, from the basic idea of portfolio replication, we assume that a bond is the asset that can be defined as

$d B(t)=r B(t) d t$

Thus, from (5), we have

$\frac{d S_{i}(t)}{S_{i}(t)}=\mu_{i} d t+\sigma_{i} d W_{i}(t)$.

Substituting (9) and (10) into (8), one has

$$
\begin{aligned}
& d P(S(t), t)=x_{1}\left(\mu_{1} S_{1}(t) d t+\sigma_{1} S_{1}(t) d W_{i}(t)\right) \\
& +x_{2}\left(\mu_{2} S_{2}(t) d t+\sigma_{2} S_{2}(t) d W_{2}(t)\right) \\
& +x_{3}\left(\mu_{3} S_{3}(t) d t+\sigma_{3} S_{3}(t) d W_{3}(t)\right)+\ldots \\
& +x_{n}\left(\mu_{n} S_{n}(t) d t+\sigma_{n} S_{n}(t) d W_{n}(t)\right)+y r B(t) d t
\end{aligned}
$$

Rearranging (11), we have

$$
\begin{aligned}
& d P(S(t), t)=\left(x_{1} \mu_{1} S_{1}(t)+x_{2} \mu_{2} S_{2}(t)\right. \\
& \left.+x_{3} \mu_{3} S_{3}(t)+\ldots+x_{n} \mu_{n} S_{n}(t)\right) d t+y r B(t) d t \\
& +\left(x_{1} \sigma_{1} S_{1}(t) d W_{1}(t)+x_{2} \sigma_{2} S_{2}(t) d W_{2}(t)\right. \\
& \left.+x_{3} \sigma_{3} S_{3}(t) d W_{3}(t)+\ldots+x_{n} \sigma_{n} S_{n}(t) d W_{n}(t)\right)
\end{aligned}
$$

By applying Ito’s lemma, we also obtain

$$
\begin{aligned}
& d P(S(t), t)=\sum_{i=1}^{n}\left[\mu_{i} S_{i}(t) P_{S_{i}}(S(t), t)\right] d t \\
& +\sum_{i=1}^{n}\left[\frac{1}{2} \sigma_{i}^{2} S_{i}(t)^{2} P_{S_{i} S_{i}}(S(t), t)\right] d t+P_{t}(S(t), t) d t \\
& +\sum_{i=1}^{n}\left[\sigma_{i} S_{i}(t) P_{S_{i}}(S(t), t) d W_{i}(t)\right] .
\end{aligned}
$$

By setting (12) equal to (13), we thus have 


$$
\begin{aligned}
& \left(x_{1} \mu_{1} S_{1}(t)+x_{2} \mu_{2} S_{2}(t)+x_{3} \mu_{3} S_{3}(t)+\ldots\right. \\
& \left.+x_{n} \mu_{n} S_{n}(t)\right)+y r B(t)=\sum_{i=1}^{n}\left[\mu_{i} S_{i}(t) P_{S_{i}}(S(t), t)\right] \\
& +\sum_{i=1}^{n}\left[\frac{1}{2} \sigma_{i}^{2} S_{i}(t)^{2} P_{S_{i} S_{i}}(S(t), t)\right]+P_{t}(S(t), t) .
\end{aligned}
$$

Also, we have

$$
\begin{aligned}
& x_{1} \sigma_{1} S_{1}(t) d W_{i}(t)+x_{2} \sigma_{2} S_{2}(t) d W_{2}(t) \\
& +x_{3} \sigma_{3} S_{3}(t) d W_{3}(t)+\ldots+x_{n} \sigma_{n} S_{n}(t) d W_{n}(t) \\
& =\sum_{i=1}^{n}\left[\sigma_{i} S_{i}(t) P_{S_{i}}(S(t), t) d W_{i}(t)\right]
\end{aligned}
$$

This yields

$x_{i}=P_{S_{i}}(S(t), t)$.

From (16), equation (7) becomes

$$
P(S(t), t)=\sum_{i=1}^{n}\left[S_{i}(t) P_{S_{i}}(S(t), t)\right]+y B(t)
$$

By rearranging, we obtain $y B(t)$ as

$$
y B(t)=P(S(t), t)-\sum_{i=1}^{n}\left[S_{i}(t) P_{S_{i}}(S(t), t)\right]
$$

From (16) and (18), the equation (14) becomes

$$
\begin{aligned}
& \sum_{i=1}^{n}\left[\mu_{i} S_{i}(t) P_{S_{i}}(S(t), t)\right]+r P(S(t), t) \\
& -\sum_{i=1}^{n}\left[r S_{i}(t) P_{S_{i}}(S(t), t)\right]=\sum_{i=1}^{n}\left[\mu_{i} S_{i}(t) P_{S_{i}}(S(t), t)\right] \\
& +\sum_{i=1}^{n}\left[\frac{1}{2} \sigma_{i}^{2} S_{i}(t)^{2} P_{S_{i} S_{i}}(S(t), t)\right]+P_{t}(S(t), t) .
\end{aligned}
$$

From (19), simplifying leads us to the final equation of

$$
\begin{aligned}
& P_{t}(S(t), t)+\sum_{i=1}^{n}\left[r S_{i}(t) P_{S_{i}}(S(t), t)\right] \\
& -r P(S(t), t)+\sum_{i=1}^{n}\left[\frac{1}{2} \sigma_{i}^{2} S_{i}(t)^{2} P_{S_{i} S_{i}}(S(t), t)\right]=0
\end{aligned}
$$

The equation (20) is the Black-Scholes equation with the extension to $n$ stochastic assets perspective. It can be seen that the equation is similar to the original Black-Scholes model; however, the extended Black-Scholes equation that we mainly propose in this paper is the generalization of assets replication which releases the limitation of the number of assets. Thus, this proposed model of the Black-Scholes equation with $n$ assets can provide the flexibility for investors in their calculations.

\section{IV. $N$ STOCHASTIC ASSETS WITH FIXED-INCOME}

From the classic model of Black-Scholes, many researchers have developed new option pricing models. The most important extended model of Black-Scholes is the BlackScholes-Merton model that utilizes the dividend factor with the original model $[3,4]$. The very first extended model of BlackScholes is derived by taking into account constant dividend. Consequently, in this paper, we apply the classical idea of option pricing model including constant dividend yield but with our proposed model of $n$ uncorrelated stochastic assets. In this case, we let $F_{i}$ represent a fixed income rate, such as dividend yield for the asset $i$ in our proposed model. Thus, we derive the Black-Scholes-Merton model under $n$ stochastic assets with fixed income by proceeding with the same method as in the previous section. As a result, we still have

$P(S(t), t)=\left(x_{1} S_{1}(t)+x_{2} S_{2}(t)+x_{3} S_{3}(t)+\ldots\right.$

$\left.+x_{n} S_{n}(t)\right)+y B(t)$

where $x_{i}$ and $y$ are the unit numbers of assets.

We obtain

$d P(S(t), t)=\left(x_{1} d S_{1}(t)+x_{2} d S_{2}(t)+x_{3} d S_{3}(t)+\ldots\right.$

$\left.+x_{n} d S_{n}(t)\right)+y d B(t)$.

and the same equation for bonds

$d B(t)=r B(t) d t$.

We can notice that (21) - (23) have no relation to fixed income. However, by considering $F_{i},(10)$ becomes

$\frac{d S_{i}(t)}{S_{i}(t)}=\left(\mu_{i}+F_{i}\right) d t+\sigma_{i} d W_{i}(t)$.

Now, we Substitute (23) and (24) into (22) to obtain

$d P(S(t), t)=x_{1}\left(\left(\mu_{1}+F_{1}\right) S_{1}(t) d t+\sigma_{1} S_{1}(t) d W_{i}(t)\right.$

$+x_{2}\left(\left(\mu_{2}+F_{2}\right) S_{2}(t) d t+\sigma_{2} S_{2}(t) d W_{2}(t)\right)$

$\left.+x_{3}\left(\mu_{3}+F_{3}\right) S_{3}(t) d t+\sigma_{3} S_{3}(t) d W_{3}(t)\right)+\ldots$

$\left.+x_{n}\left(\mu_{n}+F_{n}\right) S_{n}(t) d t+\sigma_{n} S_{n}(t) d W_{n}(t)\right)$

$+y r B(t) d t$

Rearrange (25), we get

$d P(S(t), t)=\left(x_{1}\left(\mu_{1}+F_{1}\right) S_{1}(t)+x_{2}\left(\mu_{2}+F_{2}\right) S_{2}(t)\right.$

$\left.+x_{3}\left(\mu_{3}+F_{3}\right) S_{3}(t)+\ldots+x_{n}\left(\mu_{n}+F_{n}\right) S_{n}(t)\right) d t+y r B(t) d t$

$+\left(x_{1} \sigma_{1} S_{1}(t) d W_{1}(t)+x_{2} \sigma_{2} S_{2}(t) d W_{2}(t)\right.$

$\left.+x_{3} \sigma_{3} S_{3}(t) d W_{3}(t)+\ldots+x_{n} \sigma_{n} S_{n}(t) d W_{n}(t)\right)$ 
Thus, by applying Ito's lemma and Taylor's theorem, we also obtain the following result for $d P(S(t), t)$.

$$
\begin{aligned}
& d P(S(t), t)=\sum_{i=1}^{n}\left[\mu_{i} S_{i}(t) P_{S_{i}}(S(t), t)\right] d t+ \\
& +\sum_{i=1}^{n}\left[\frac{1}{2} \sigma_{i}^{2} S_{i}(t)^{2} P_{S_{i} S_{i}}(S(t), t)\right] d t+P_{t}(S(t), t) d t \\
& +\sum_{i=1}^{n}\left[\sigma_{i} S_{i}(t) P_{S_{i}}(S(t), t) d W_{i}(t)\right]
\end{aligned}
$$

Since (26) is equal to (27), we obtain

$$
\begin{aligned}
& \left(x_{1}\left(\mu_{1}+F_{1}\right) S_{1}(t)+x_{2}\left(\mu_{2}+F_{2}\right) S_{2}(t)\right. \\
& \left.+x_{3}\left(\mu_{3}+F_{3}\right) S_{3}(t)+\ldots+x_{n}\left(\mu_{n}+F_{n}\right) S_{n}(t)\right) \\
& +y r B(t)=\sum_{i=1}^{n}\left[\mu_{i} S_{i}(t) P_{S_{i}}(S(t), t)\right] \\
& +\sum_{i=1}^{n}\left[\frac{1}{2} \sigma_{i}^{2} S_{i}(t)^{2} P_{S_{i} S_{i}}(S(t), t)\right]+P_{t}(S(t), t)
\end{aligned}
$$

with the condition

$$
\begin{aligned}
& x_{1} \sigma_{1} S_{1}(t) d W_{i}(t)+x_{2} \sigma_{2} S_{2}(t) d W_{2}(t) \\
& +x_{3} \sigma_{3} S_{3}(t) d W_{3}(t)+\ldots+x_{n} \sigma_{n} S_{n}(t) d W_{n}(t) \\
& =\sum_{i=1}^{n}\left[\sigma_{i} S_{i}(t) P_{S_{i}}(S(t), t) d W_{i}(t)\right]
\end{aligned}
$$

From (29), we obtain

$$
x_{i}=P_{S_{i}}(S(t), t) \text {. }
$$

From (30), equation (7) becomes

$$
P(S(t), t)=\sum_{i=1}^{n}\left[S_{i}(t) P_{S_{i}}(S(t), t)\right]+y B(t)
$$

Then, we have

$$
y B(t)=P(S(t), t)-\sum_{i=1}^{n}\left[S_{i}(t) P_{S_{i}}(S(t), t)\right]
$$

From (30) and (32), the equation (28) becomes

$$
\begin{aligned}
& \sum_{i=1}^{n}\left[\left(\mu_{i}+F_{i}\right) S_{i}(t) P_{S_{i}}(S(t), t)\right]+r P(S(t), t) \\
& -\sum_{i=1}^{n}\left[r S_{i}(t) P_{S_{i}}(S(t), t)\right]=\sum_{i=1}^{n}\left[\mu_{i} S_{i}(t) P_{S_{i}}(S(t), t)\right] \\
& +\sum_{i=1}^{n}\left[\frac{1}{2} \sigma_{i}^{2} S_{i}(t)^{2} P_{S_{i} S_{i}}(S(t), t)\right]+P_{t}(S(t), t) .
\end{aligned}
$$

By simplification, we have

$$
\begin{aligned}
& P_{t}(S(t), t)+\sum_{i=1}^{n}\left[\left(r-F_{i}\right) S_{i}(t) P_{S_{i}}(S(t), t)\right] \\
& -r P(S(t), t)+\sum_{i=1}^{n}\left[\frac{1}{2} \sigma_{i}^{2} S_{i}(t)^{2} P_{S_{i} S_{i}}(S(t), t)\right]=0
\end{aligned}
$$

In (33) and (34), we have derived an extended model of BlackScholes-Merton's with $n$ stochastic assets and fixed income. In comparison, the equation (34), being the Black-ScholesMerton model with the proposed extension of $n$ stochastic assets perspective with fixed income, is very similar to the original Black-Scholes-Merton equation but incorporates the $n$ stochastic assets assumption. This extension may lead to more general applications based on the assumption of uncorrelated assets.

\section{APPLICATIONS}

The proposed models in this paper represent another aspect in the derivation of Black-Scholes-Merton model taking into account $n$ stochastic assets as well as Black-Scholes-Merton model under the environment of $n$ stochastic assets with fixed income such as dividend yield. In the real world application, these extended models significantly affect to the actual investment calculations and financial analyses. Both the classical Black-Scholes model without dividend yield factor, and the well-known Black-Scholes-Merton model with dividend yield are the outstanding tools that can help investors to accurately estimate the value of derivative pricing based on one underlying asset. As a simple example, the investor can calculate the fair value of an option by using the information of underlying stocks. Nevertheless, in some other situations, there might be some financial unit prices that rely on more than one asset. For example, sometimes the value of a business may be mathematically valuated by cash plus real estate value and both cash and real estates are certainly based on bonds or interest rates. In this case, if you are an investor, you need to analyze deeply into the fair value of the business. The proposed Black-Scholes-Merton model with $n$ stochastic assets may help to satisfy the needs of this investor. Another example of applications is in the valuation of current insurance needs. Usually, the basic way to calculate insurance needs is to find the difference between the present value of the required capital and the current assets value. Analytically, our proposed formulations of the Black-Scholes-Merton model with $n$ stochastic assets with dividend could potentially help in making a decision to finalize the number of insurance needs, since the required capital and current assets value should be calculated by having more than one asset and some assets have dividend returns. Thus, in conclusion, our proposed model can be applied in various ways depending on the suitability of the situation.

\section{CONCLUSION}

We have discussed the derivation of the Black-Scholes model without dividend yield consideration and also the Black-Scholes-Merton model with the dividend yield factor. Both models can be derived by the method of portfolio replication. In this paper, we propose both of the BlackScholes model and the Black-Scholes-Merton model under the environment of $n$ underlying stochastic assets following the Geometric Brownian Motion. Finally, we extend the model by 
taking into account the fixed income. In this way, the BlackScholes-Merton model can be applied in some more complicated situations such as a situation that an option pricing depends on many stochastic assets with fixed dividend. This implies that in future work, in order to develop extensions of the model for more varied cases, the stochastic parameters should be investigated such as stochastic dividends or stochastic volatility.

\section{ACKNOWLEDGMENT}

Appreciation is extended toward Mahidol University, and the Centre of Excellence in Mathematics, CHE, Thailand, for all supports.

\section{REFERENCES}

[1] F. Black and M. Scholes, "The Pricing of Options and Corporate Liabilities," Journal of Political Economy, Vol.81, No.3, 1973, pp. 637-654.

[2] N. A. Chriss, "Black-Scholes and Beyond Option Pricing Models," Irwin Professional Publishing, USA, 1997.

[3] R. C. Merton, "Theory of Rational Option Pricing," The Bell Journal of Economics and Management Science, Vol.4, No.1, 1973, pp. 141183.

[4] R. C. Merton, "Option Pricing when Underlying Stock Returns Are Discontinuous," Journal of Financial Economics, Vol.3, No.1-2, 1976, pp. 125-144.

[5] M. Kishimoto, "On the Black-Scholes Equation: Various Derivations." MSE Term Paper, 2008.

[6] M. J. Stutzer, "Simple Entropic Derivation of a Generalized BlackScholes Option Pricing Model," Entropy, Vol.2, No.2, 2000, pp. 7077.
Creative Commons Attribution License 4.0 (Attribution 4.0 International, CC BY 4.0)

This article is published under the terms of the Creative Commons Attribution License 4.0

https://creativecommons.org/licenses/by/4.0/deed.en_US 